\title{
High performance PA6/CNTs nanohybrid fibers prepared in the melt
}

\author{
R. Scaffaro ${ }^{\mathrm{a}, *}$, A. Maio $^{\mathrm{a}}$, A.C. Tito ${ }^{\mathrm{b}}$ \\ ${ }^{a}$ Department of Civil, Environmental, Aerospace, Materials Engineering, University of Palermo, Viale delle Scienze, Ed. 6, 90128 Palermo, Italy \\ ${ }^{\mathrm{b}}$ Department of Chemistry, Materials and Chemical Engineering 'G. Natta', Politecnico di Milano, Piazza L. da Vinci 32, 20133 Milano, Italy
}

\section{A R T I C L E I N F O}

\section{Article history:}

Received 16 May 2012

Received in revised form 27 July 2012

Accepted 16 August 2012

Available online 29 August 2012

\section{Keywords:}

A. Carbon nanotubes

B. Electrical properties

C. Elastic properties

D. Raman spectroscopy

E. Melt-spinning

\begin{abstract}
A B S T R A C T
Commercial and home-made carbon nanotubes (CNTs) were plasma treated under oxygen atmosphere and then added to polyamide 6 (PA6) in order to prepare fibres by melt spinning. For comparison, pristine nanofillers were used too. The effect of functionalization and of filler characteristics on the morphological, rheological, mechanical and electrical properties of the fibres was studied by TEM and SEM, rheological measurements, tensile and electrical conductivity tests. The results demonstrated that the functionalization led to a better mechanical performance and the morphological analysis confirmed that the adhesion, the dispersion and the alignment of the nanotubes within the polymer matrix were improved when using functionalized CNTs. Electrical tests marked that functionalization slightly reduced the conductivity of the materials.
\end{abstract}

(c) 2012 Elsevier Ltd. All rights reserved.

\section{Introduction}

In the recent years, the attention of both industry and academia was attracted by the possibility to conjugate good mechanical performance and electrical conductivity in common thermoplastic polymers. In particular, the primary aim is to obtain new materials bearing the flexibility and the low density typical of polymers with the electrical conductivity typical of metals or semiconductors in the view of applications in sensors, electronics, electromagnetic shields or antistatic devices [1,2].

Intrinsic conductive polymers (ICPs) may represent a valid solution to achieve this goal but they are often difficult to process and display low ductility, low thermal stability and low aging resistance. Some attempts to combine a flexible support with ICPs were also investigated but, despite some positive results, the control of the process appeared difficult and requested the presence of a solvent [3].

Undoubtedly, the most attractive method to produce electrically conductive polymer-based materials is adding appropriate fillers which are able to provide the desired electrical properties $[1,2,4]$. The effectiveness of this operation lies in a good dispersion of the filler with the primary goal to get the highest electrical conductivity with the lowest filler content. Using nanoscaled fillers offers the possibility to achieve the aim by adding small amounts (even less than $1 \mathrm{wt} . \%$ ) due to their high specific surface. Among these nanofillers, CNTs are interesting because of their unique

\footnotetext{
* Corresponding author.

E-mail address: roberto.scaffaro@unipa.it (R. Scaffaro).
}

combination of low density, large aspect ratio, good dimensional stability, high mechanical performance and electrical conductivity $[1,2,4,5]$. The ways to add CNTs into polymer matrices refer to two different inclusion approaches: in solution and in the melt. Methods bearing to the first category allow achieving the best results in terms of nanofiller dispersion but they are expensive and may potentially cause problems in solvent removal/recycle. On the other hand, melt processing allows to reduce the costs and to speed up the manufacturing operations but it is more difficult to avoid CNTs bundling and to improve the interfacial adhesion with polymer matrices. The functionalization of CNTs is an effective way to prevent nanotube aggregation, which helps to better disperse and stabilize the CNTs within a polymer matrix. Typically, modified CNTs include specific functional moieties (e.g. carboxyls, amine, hydroxyls, etc.) introduced by dedicated processes [5-10].

Volpato et al. [11] prepared electrospun fibres of polyamide 6 (PA6) reinforced with carboxylated CNTs to be used as scaffold for cells proliferation. Deng et al. [12] evaluated the carboxylation of CNTs on the mechanical properties of PA6/CNTs nanocomposites, finding higher values of the modulus and of the properties at break, showing increased strength in the range of 5-10 MPa. Zhou et al. [13] added amino modified CNTs to PA6, observing a drastic increase of the mechanical performance (up to 200\%) together with the growth of shish-kebab PA6 structures onto the CNT surface.

Dintcheva et al. [14] studied the effect of the elongational flow on the morphological and mechanical properties of PA6/CNTs fibres finding that adding CNTs did not cause significant changes. Also in this case, a shish-kebab morphology of PA6 grown onto CNTs walls was observed. 
According to our previous works [5,10], in this paper we chose to functionalize, in oxygen plasma, two samples of CNTs that differ for synthesis method and characteristics in order to introduce polar groups on the nanotubes walls thus promoting the adhesion with polyamide 6 . The CNTs were then added to PA6 by melt mixing and fibres were subsequently obtained by melt spinning and characterized from a mechanical, morphological and electrical point of view, comparing the properties of composites prepared with neat or functionalized CNTs.

\section{Experimental}

\subsection{Materials}

Some of the carbon nanotubes used in the present work were a sample of commercial MWCNTs (CNT 1, Sigma Aldrich) produced by arc-discharge and having a length in the range $5-9 \mu \mathrm{m}$ with a diameter in the range $100-170 \mathrm{~nm}$ and a purity $>90 \%$. For comparison, home-made CNTs (CNT 2) were produced by fluidized bed catalytic chemical vapour deposition (FBCCVD) of ethylene using alumina-supported iron as catalyst as described in $[15,16]$. In brief, a gas mixture of ethylene, hydrogen and nitrogen was used as carbon source and fluidizing gases; $\gamma$-alumina particles, previously treated by wet impregnation to deposit iron $(10 \% \mathrm{w} / \mathrm{w})$, were employed as catalytic powders. The process productive capacity of CNTs is $8.3 \mathrm{~g} / \mathrm{min}$.

The control of the fluidization quality is of major importance to operate in isothermal conditions and to obtain the homogeneous mixing of the solid particles: the fluidizations ratio $U / U_{m f}$ (where $U$ is the gas flow velocity and $U_{m f}$ the minimum fluidization velocity) was, for this reason, set to about 16 . The nominal temperature of the process was $650^{\circ} \mathrm{C}$. The process selectivity in terms of MWCNTs was close to 100\% (no amorphous carbon was detected), with an ethylene conversion always greater than $85 \%$. The composite material obtained from the FBCCVD production step, consisting of nanotubes, iron and alumina, was purified by a sulphuric acid treatment in order to accomplish the total dissolution of alumina and the partial elimination of the iron contained in the tubes. After
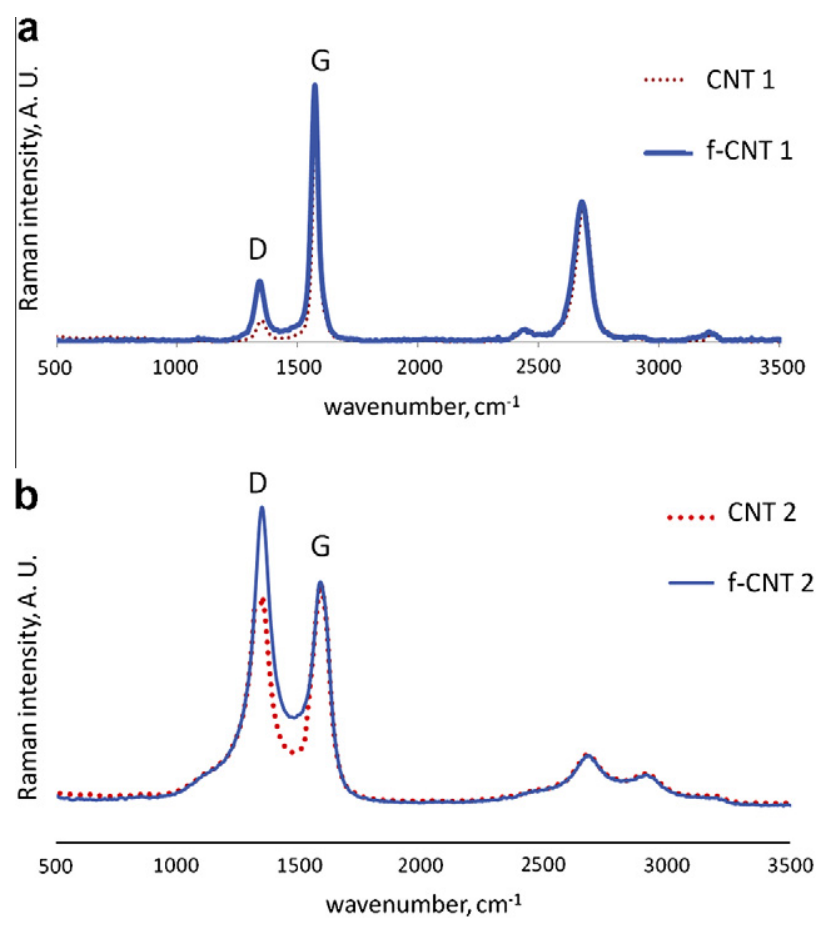

Fig. 1. Raman spectra of neat and functionalized CNT 1 (a) and CNT 2 (b).

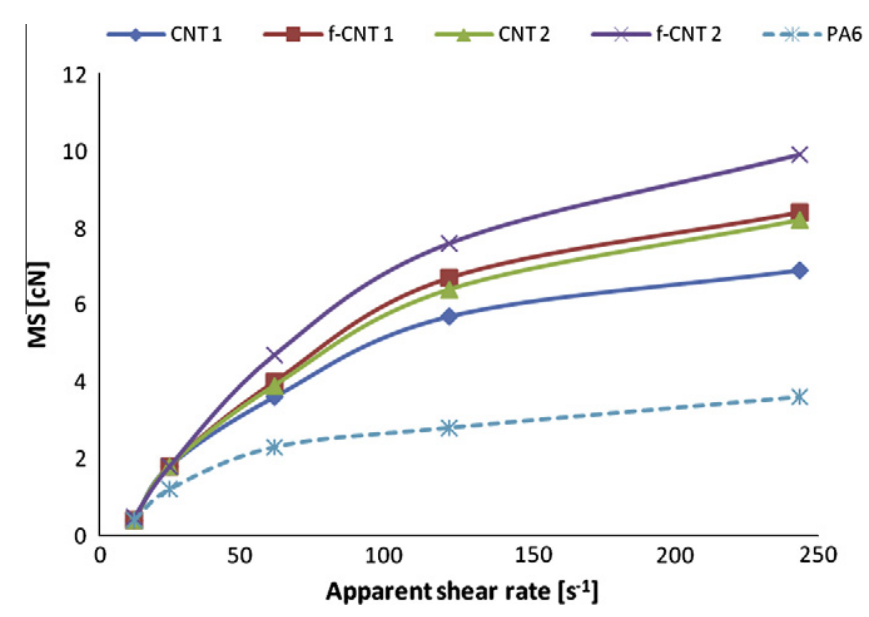

Fig. 2. MS as a function of apparent shear rate for PA6 and for the composites containing CNTs.

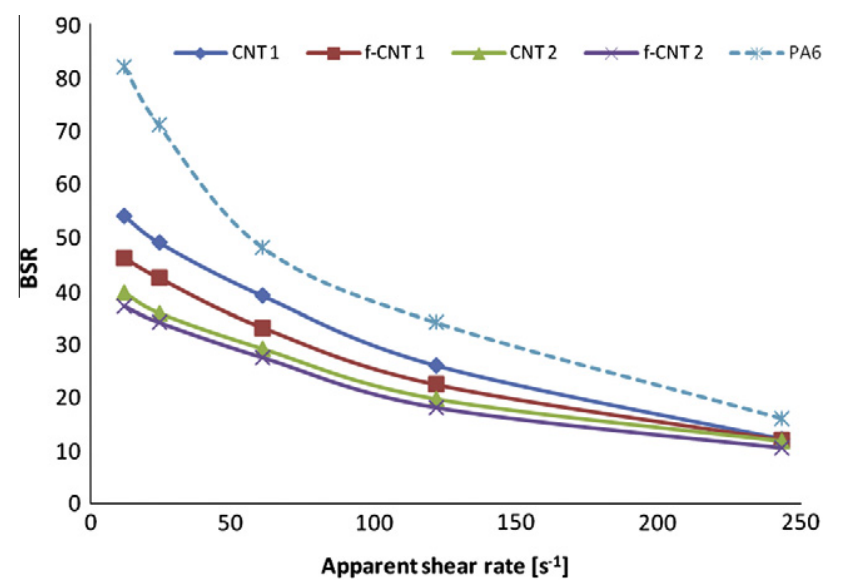

Fig. 3. BSR as a function of apparent shear rate for PA6 and for the composites containing CNTs.

this latter treatment, CNTs exhibited purity $>99 \%$, their average length ranged from 1 to $5 \mu \mathrm{m}$, their inner diameter ranged from 3 to $10 \mathrm{~nm}$ and their outer diameter ranged from 6 to $19 \mathrm{~nm}$. The average aspect ratio was approximately 40 for CNT 1 and over 300 for CNT 2. More details on the morphology of the two samples of CNTs can be found in the supporting information section.

The PA6 adopted in this work was a commercial sample of Radilon S35 F 100 NAT, supplied by Radicinova (Italy). It has an inherent viscosity measured in sulfuric acid of $2.05 \mathrm{dl} / \mathrm{g}$. In order to avoid hydrolytic scission during processing, it was dried overnight under vacuum at $120^{\circ} \mathrm{C}$.

\subsection{Plasma functionalization}

Both samples of CNTs were treated in a plasma reactor with radiofrequency polarized anode (Gambetti, Italy). The nanotubes were dried under vacuum at $120^{\circ} \mathrm{C}$ overnight, fractionated in a mortar, put on a watch glass and then fed to the reactor, using oxygen as gas, a power of $120 \mathrm{~W}$ and a treatment time of $10 \mathrm{~min}$, according to our previous studies $[5,10]$.

\subsection{Fibres preparation}

Composites containing 2 wt.\% of CNTs were prepared in a batch mixer (Brabender PLE330) equipped with a mixing chamber of 

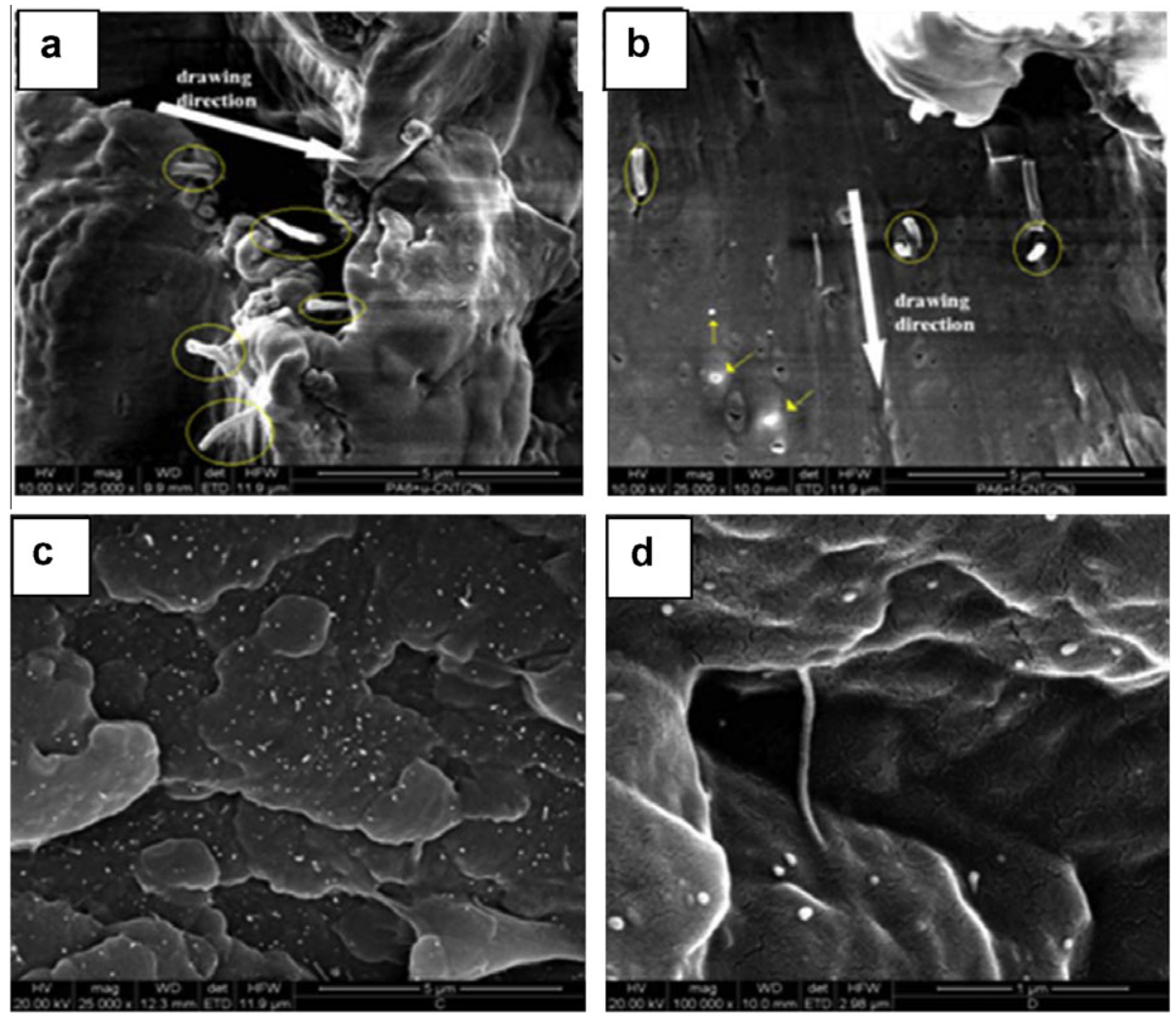

Fig. 4. SEM micrographs of PA6/CNT 1 (a), PA6/f-CNT 1 (b), PA6/CNT 2 (c) and PA6/f-CNT 2 (d),

$50 \mathrm{~cm}^{3}$. The temperature was $240{ }^{\circ} \mathrm{C}$, the rotational speed was $64 \mathrm{rpm}$ and the processing time about $5 \mathrm{~min}$, enough to achieve a constant value of the mixing torque. In detail, the polymer and the CNTs, premixed in the solid state, were fed to the mixer at a rotational speed of $20 \mathrm{rpm}$. This operation last no longer than $1.5 \mathrm{~min}$. Once closed the chamber, the rotational speed was raised to the final value of $64 \mathrm{rpm}$. At the end of processing, the material fed out the mixer was collected within $2 \mathrm{~min}$, the remainder was discarded.

The blend was then ground in pellets $1-2 \mathrm{~mm}$ sized and fed to a capillary viscometer (CEAST Rheoscope 1000) equipped with a spinning unit and preheated at $240^{\circ} \mathrm{C}$. The barrel speed was set to $5 \mathrm{~mm} / \mathrm{min}$ while the collecting pulley speed was set to $10 \mathrm{rpm}$ in order to get fibres with a constant diameter of approximately $50-60 \mu \mathrm{m}$.

\subsection{Characterizations}

The functionalization level of the CNTs was followed by microRaman spectroscopy at room temperature on a Bruker-Senterra micro-Raman equipped with a $532 \mathrm{~nm}$ diode laser excitation and $20 \mathrm{~mW}$ power. Nonconfocal measurements were carried out in the range $4000-400 \mathrm{~cm}^{-1}$ with a spectral resolution between 9 and $15 \mathrm{~cm}^{-1}$.

In order to investigate the spinability of the PA6/CNTs nanocomposites, the melt strength (MS) and the breaking stretching ratio (BSR) as a function of the apparent shear rate were measured for all the materials on the same equipment used for the fabrication of the fibres. The barrel speed ranged from 1 to $20 \mathrm{~mm} / \mathrm{min}$ and the collecting pulley acceleration was set to $100 \mathrm{rpm} / \mathrm{min}$.

The tensile mechanical properties of the fibres were measured using an Instron 3365 dynamometer. The grip distance was $30 \mathrm{~mm}$ while the tensile speed was $1 \mathrm{~mm} / \mathrm{min}$ for the first $2 \mathrm{~min}$ and $100 \mathrm{~mm} / \mathrm{min}$ thereafter. The results here reported are the average of at least 10 measurements.
In order to study the dispersion/adhesion of the fibres in the nanocomposites, scanning electron microscopy (SEM) was performed using a SEM FEI Quanta 200 ESEM on the fractured surfaces of the samples after tensile tests, sputter-coated with a thin layer of gold to avoid electrostatic charging under the electron beam. In addition, to study more in detail the morphology of the nanotubes, the PA6/CNTs interface and to study the eventual alignment of the nanotubes under the elongational flow, transmission electron microscopy (TEM) tests were performed at the CGA-Centro Grandi Apparecchiature, University of Palermo, by using a Jeol 2100 apparatus. The fibres were immersed in epoxy resin and, after solidification, cut by using an ultra-cryo-microtome in slices $100 \mathrm{~nm}$ thick. The slices were put on a $3 \mathrm{~mm}$ copper grid with a lacey carbon film and then analyzed under an acceleration voltage of $200 \mathrm{kV}$.

Electrical conductivity tests were carried out using a Keithley 2612 multimeter equipped with a four probe cell. The resistance data collected on the nanocomposite fibres were then used to calculate the electrical conductivity.

\section{Results and discussion}

Raman spectroscopy measurements reported in Fig. 1 were carried out on five different zones for each sample and averaged. ANOVA analysis, here not detailed for sake of brevity, detected no significant differences between the experimental data for each sample. The resulting spectra have been then elaborated by subtracting the baseline and normalized with respect to the $G$ band of CNTs, in order to allow the analysis of the differences between neat and functionalized samples by comparing, for each sample, the related ratio between the intensities of D-band and G-band (ID/IG ratio). This ratio can be correlated with the degree of functionalization by the different vibration mode of carbon atoms in the presence of defects, generated during the functionalization process and associated to the presence of oxygen or other atoms 

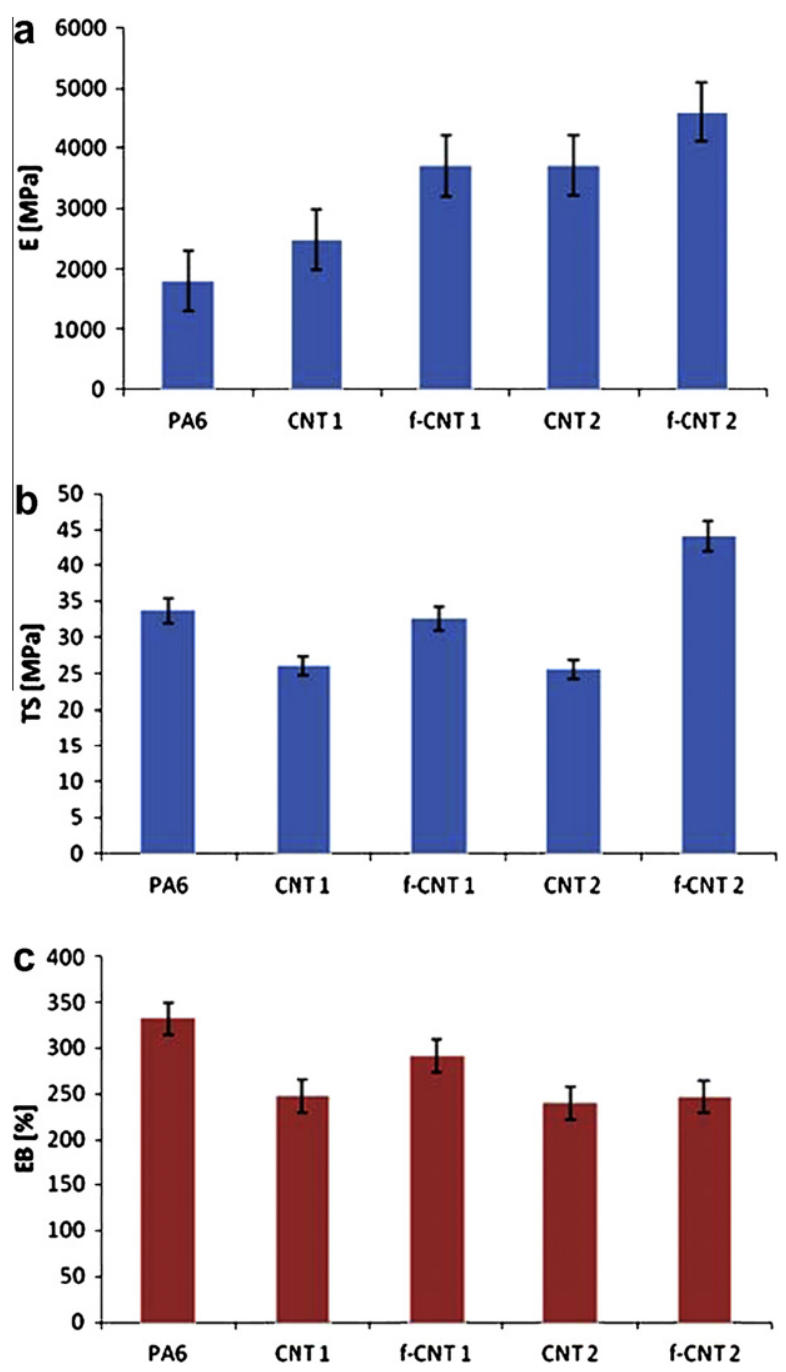

Fig. 5. Elastic modulus (a), tensile strength (b) and elongation at break (c) of neat PA6 and of composites containing CNTs.

in the nanotube structure [17-19]. In particular, the G-band is associated to the stretching of $\mathrm{C}-\mathrm{C}$ bonds in graphitic materials ( $\mathrm{sp}^{2}$ hybridization) while the D-band is associated with phonons close to the boundary of the Brillouin zone, usually detectable by Raman for disordered crystals (defects in $\mathrm{sp}^{2}$ carbons). An increase of this ratio can be correlated with the degree of functionalization by the different vibration modes of carbon atoms in the presence of defects, generated during the functionalization process and associated to the presence of oxygen or other atoms in the nanotube structure. $[5,17,18]$.

In Fig. 1 there are reported the spectra of neat and functionalized CNT 1, Fig. 1a, and CNT 2, Fig. 1b. The ID/IG ratio in pristine CNT 1, 0.08, is sensibly lower than that observed in CNT 2, 0.994. This can be explained considering that CNT 2 were deeply purified in sulphuric acid that induces structural defects on the sidewalls and cuts CNTs tips [19,20], introducing, at the same time, oxygen moieties, whose vibration is associated to the $\mathrm{D}$ band. After plasma treatment, both samples show an increase of the ID/IG ratio, with a sharp increase of the $\mathrm{D}$ band with a corresponding increase of the ID/IG ratio that becomes 0.22 for CNT 1 and 1.354 for CNT 2. This result confirms that relevant changes occurred in the nanotube structure during the plasma treatment.

Another indirect evidence of the introduction of polar moieties onto the CNTs surface can be found in the analysis of the rheological behaviour under elongational flow.
In Figs. 2 and 3 there are reported, respectively, the melt strength (MS) and the breaking stretching ratio (BSR) as a function of the apparent shear rate for PA6 and for the composites containing both samples of neat and functionalized CNTs. Adding CNTs to PA6 causes a sharp increase of MS even if some differences must be put into evidence. Generally, the increments of MS are more intense when CNT 2 are added and when functionalized samples are used. Therefore, the highest increase of MS is observed for the systems PA6/f-CNT 2 while the lowest is observed in the system PA6/CNT 1. These results can be interpreted considering that the presence of polar oxygen moieties in the functionalized CNT 1 and CNT 2 (f-CNT 1 and f-CNT 2 respectively) is responsible of a better adhesion between the nanofiller and the matrix. This higher interaction between the two phases causes an increase of the elongational viscosity and, eventually, an increase of the melt strength. In this sense, f-CNT $2>$ CNT $2 \approx \mathrm{f}-\mathrm{CNT} 1>\mathrm{CNT} 1$. In order to comment this finding, it can be observed that there are two main concurrent effects affecting MS: (i) L/D ratio (and, more generally, morphology) of the CNTs and (ii) adhesion of CNTs to the matrix. In this case, it can be speculated that the effect of the worse morphology of CNT 1 in terms of adhesion and dispersion are somehow counterbalanced by the higher adhesion induced by their functionalization. This feature leads to behaviour very similar to that of CNT 2 that are not functionalized but with a better morphology. For the same reason, it is clear that the best performance is found in the f-CNT 2 containing materials and the worst when unfunctionalized CNT 1 are used.

As regards BSR, the situation is just the opposite. The highest value, i.e. the highest deformability, is reported by PA6, while the CNTs containing systems are progressively less deformable when passing from CNT 1 to f-CNT 2. To explain these results, it must be invoked again the better adhesion when functionalized CNTs are used. This may cause the alignment of the CNTs along the flow direction that increase the rigidity of the material. In order to go deeper inside this matter, tensile mechanical testing and morphological analysis were performed on all the materials.

The SEM micrographs of the fractured surface of the PA6 fibres containing neat and functionalized CNTs are reported in Fig. 4. In PA6/CNT 1 fibres, Fig. 4a, it can be noted the orientation of the fibres with respect to the drawing direction. In addition, there are several nanotubes pulled out the matrix and holes left by others dewetted and expelled during the cryogenic fracture. Moreover, it is well visible an aggregate of bundled nanotubes that were not dispersed during processing. These latter features suggest that the dispersion and the adhesion/interaction with the matrix are globally poor. The situation is different when CNT 2 are used, Fig. 4c. In this case the dispersion is better as the nanotubes are uniformly dispersed. Even the adhesion appears improved as no pulled out nanotubes can be detected. This occurrence can be explained considering that, the oxygen moieties introduced during the purification process increased the polarity of the CNTs and, consequently, their interaction with the PA6 matrix, thus allowing a better dispersion and adhesion.

Plasma treatment further improves the PA6/CNTs interactions. In PA6/f-CNT 1, Fig. 4b, the nanotubes appear more adherent and oriented along the drawing direction. Moreover, no bundles are visible and the CNTs appear well separated each other. The best performance, however, is shown by the f-CNT 2 containing system, Fig. $4 \mathrm{~d}$. In this case, in fact, there is not only a very good adhesion and dispersion of the nanotubes, but it is also possible to find isolated nanotubes bridging the cracks edges, evidencing an excellent adhesion between these CNTs and the PA6.

These observations find confirmation in the mechanical performance of the fibres. In Fig. 5a-c there are reported the tensile mechanical properties of all the fibres prepared in the frame of this work. 

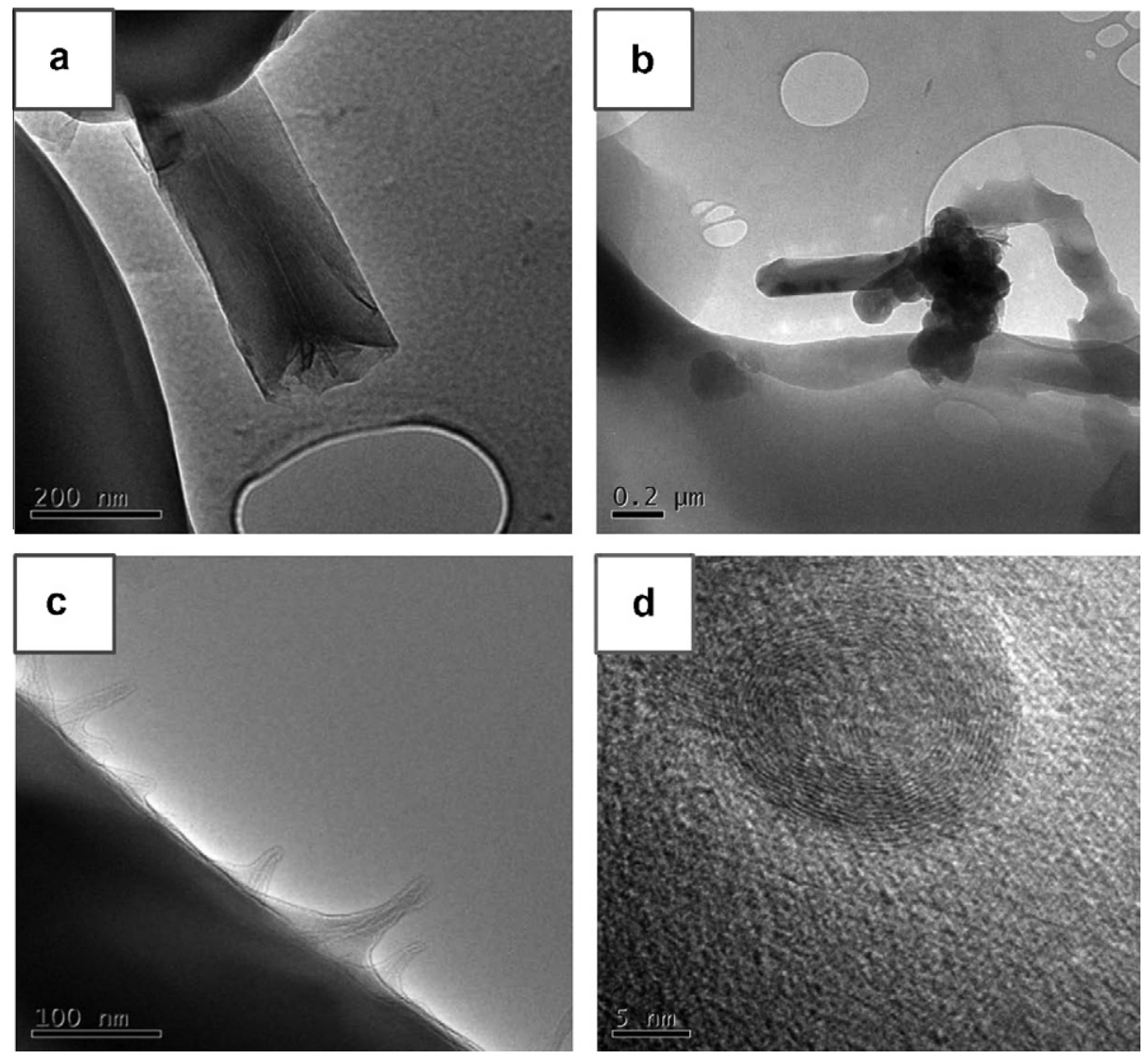

Fig. 6. TEM micrographs PA6/CNT 1 (a), PA6/f-CNT 1 (b) and PA6/f-CNT 2 (c-d).

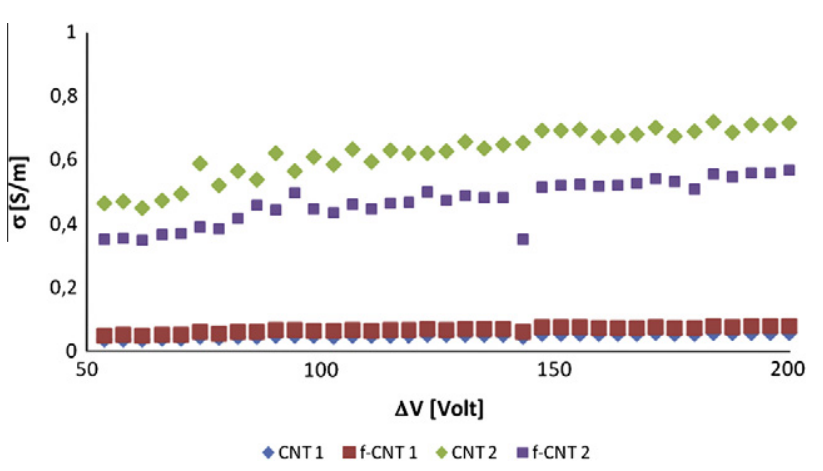

Fig. 7. Electrical conductivity as a function of the potential for PA6 based nanocomposite fibres.

Adding CNTs or $\mathrm{f}-\mathrm{CNTs}$ causes in all the cases an increase of the elastic modulus, Fig. 5a. In particular the best results are shown by the system containing f-CNT 2 whose modulus is about three times higher than that of neat PA6. In addition and as expected, it is well evident that the composites containing CNT 2 show higher moduli than those containing CNT 1 and functionalized CNTs are better than neat ones, following the same trend already observed and commented for MS. As regards TS, Fig. 5b, the materials containing neat CNTs show values slightly lower than pure PA6. PA6/f-CNT 1 shows values comparable with PA6 while PA6/f-CNT 2 has values sensibly higher with respect to the pure matrix. The better performance of the materials containing functionalized CNTs can be explained considering that the better adhesion matrix/nanofiller enhances the stress transfer between the two phases thus improving the ultimate resistance of the composites. As expected, EB decreases for all the materials and this is according to the reduced deformability already observed and commented about BSR. In this case, however, the composites display a very similar behaviour.

A deeper investigation that confirmed the stronger interaction between functionalized CNTs and PA6 was performed by TEM analysis. In PA6/CNT 1, Fig. 6a, it is possible to identify a single nanotube with smooth surface, evidencing the absence of any adhesion with the matrix. The morphology of PA6/f-CNT 1, Fig. 6b, is different from that observed in the material containing CNT 1 as the nanotubes are partially covered with PA6, indicating a higher affinity between the two components. The adhesion is further improved when using f-CNT 2, Fig. 6c-d. In this case, in fact, it is possible to see several isolated nanotubes that are wrapped into PA6 that completely adhere to their surface. This higher affinity between the matrix and CNTs allows their better orientation, as evidenced by the preferential alignment along the drawing direction.

The different characteristics of the nanotubes have also consequences on the electrical properties of the materials. In Fig. 7 it is reported the electrical conductivity as a function of the potential measured on the PA6 based nanocomposite fibres. CNT 1 containing materials show the worst conductivity, very close to that measured for the pure matrix (below the detection sensitivity of the instrument, i.e. $<10^{-15} \mathrm{~S} / \mathrm{m}$ ). Moreover, there is practically no difference between using neat and functionalized nanotubes and this can be ascribed to the low aspect ratio of these CNTs samples and to their globally bad dispersion into the matrix. Under this conditions and at the CNT level chosen (2 wt.\%) the fibres are not conductive. The situation is different for the CNT 2 containing systems. In this case, the fibres show a weak increment of conductivity. This can be explained considering the higher aspect ratio with respect to CNT 1 and higher orientation that, likely, allowed the percolation of the nanotubes and, consequently, the increase 
of the electrical conductivity, also according with other authors $[21,22]$. Surprisingly, the plasma functionalization causes a slight decrease of this property, despite the higher level of adhesion, dispersion and orientation achieved, as demonstrated by the above presented data. This unexpected result can be explained considering that the functionalization partially destroyed the graphenic $\mathrm{sp}^{2}$ structure of the CNTs walls after introducing oxygen moieties as demonstrated by the increase of the ID/IG ratio as measured by Raman spectroscopy and discussed in our previous works $[5,10]$. Other authors [23-25] while studying PA12/CNT systems found some relationship between the rheological behaviour of PA12, its end groups and the electrical conductivity of the nanocomposites. Another possible explanation of the phenomenon can also be found in the high polymer/nanotubes interactions: the polymer, completely wrapping the nanotubes, can considerably reduce the electron tunnelling that is at the base of the electrical conductivity in this kind of systems $[21,22,26]$.

These aspects would deserve a deeper analysis but, being beyond the aim of the paper, they were not investigated in this work and will be developed in further investigations.

\section{Conclusions}

In this work, a commercial (CNT 1) and home-made sample of carbon nanotubes (CNT 2), were functionalized by plasma treatment under oxygen atmosphere, added ( $2 \mathrm{wt} . \%)$ to polyamide 6 (PA6) and melt spun. Adding functionalized CNTs (f-CNTs) causes an increase of melt strength, coupled with a decrease of deformability. TEM and SEM morphological analyses confirmed that f-CNTs have a better dispersion and adhesion with the PA6 matrix. Moreover, they appear more oriented along the drawing direction if compared with the unfunctionalized ones. This was attributed to the presence of polar moieties introduced during the functionalization as confirmed by the sharp increase of the ID/IG ratio measured by Raman spectroscopy.

Between the two CNTs samples, CNT 2 showed the overall best performance from a mechanical and electrical point of view. The materials containing f-CNTs show the highest moduli and ultimate resistance. It must be highlighted that both functionalization and processing (in particular the elongational flow adopted to prepare the fibres) co-operate in achieving the enhancement of the mechanical performance.

The electrical conductivity reduces when f-CNTs are used. This can be explained invoking the partial disruption of the graphenic structure of the CNTs walls by the introduction of oxygen moieties and by the wrapping of PA6 onto f-CNTs that inhibits electron tunnelling thus counterbalancing the benefits of the better dispersion and alignment.

The results suggest that the mechanical and electrical performance can be improved when a better dispersion/unbundling of the CNTs is obtained. This can be achieved by controlling the functionalization level and the quality of the starting nanotubes.
Finally, this method combining solventless modification of CNTs and melt processing to produce PA6/CNTs nanocomposites can be easily scaled-up to industrial production due to the shorter times and the reasonably lower costs if compared with solvent based manufacturing.

\section{Appendix A. Supplementary material}

Supplementary data associated with this article can be found, in the online version, at http://dx.doi.org/10.1016/j.compscitech. 2012.08.010.

\section{References}

[1] Li C, Liang T, Lu W, Tang C, Hu X, Cao M, et al. Compos Sci Technol 2004;64(1314):2089-96.

[2] Avouris P, Hertel T, Martel R, Schmidt T, Shea HR, Walkup RE. Carbon nanotubes: nanomechanics, manipulation, and electronic devices. Appl Surf Sci 1999;141:201-9.

[3] Scaffaro R, Lo Re G, Dispenza C, Sabatino MA, Armelao L. A new route for the preparation of flexible skin-core poly(ethylene-co-acrylic acid)/polyaniline functional hybrids. React Funct Polym 2011;71(12):1177-86.

[4] Vaisman L, Wagner HD, Marom G. Adv Colloid Interface Sci 2006;121123:37-46.

[5] Scaffaro R, Maio A, Agnello SP, Glisenti A. Plasma Process Polym 2012;9(5):503-12.

[6] Spitalsky Z, Tasis D, Papagelis K, Galiotis C. Prog Polym Sci 2010;35(3):357-401.

[7] Grossiord N, Miltner HE, Loos J, Meuldijk J, Mele BV, Koning. Chem Mater 2007;19(15):3787-92.

[8] Georgakilas V, Kordatos K, Prato M, Guldi DM, Holzingger M, Hirsch A. J Am Chem Soc 2002;124(5):760-1.

[9] Yook JY, Jun J, Kwak S. Appl Surf Sci 2010;256(23):6941-4.

[10] Scaffaro R, Maio A. Enhancing the mechanical performance of polymer based nanocomposites by plasma-modification of nanoparticles. Polym Test 2012;31(7):889-94.

[11] Volpato FZ, Ramos SLF, Motta A, Migliaresi C. J Bioact Compat Polym 2011;26:35-47.

[12] Deng H, Bilotti E, Zhang R, Wang K, Zhang Q Peijs T, et al. J Appl Polym Sci 2011;120(1):133-40.

[13] Zhou Q, Hu YC, Sun XN, Weng YJ. Adv Mater Res 2011;462:287-90.

[14] Dintcheva NT, Arrigo R, Morreale M, La Mantia FP, Matassa R, Caponetti E. Polym Adv Technol 2011;22(12):1612-9.

[15] Corrias M, Caussat B, Aynal A, Durenol J, Kihn Y, Kalck P, et al. Carbon nanotubes produced by fluidized bed catalytic CVD: first approach of the process. Chem Eng Sci 2003;58(19):4475-82.

[16] Mazzocchia CV, Bestetti M, Acierno D, Tito A. A process for the preparation of a catalyst, a catalyst obtained thereby, and its use in the production. European Patent 2213369 (A1); 2010-08-04.

[17] Li CC, Lin JL, Huang SJ, Lee JT, Chen CH. Colloids Surf A: Physicochem Eng Aspects 2007;297(1-3):275.

[18] Lehman JH, Terrones M, Mansfield E, Hurst KE, Meunier V. Carbon 2011;49:2581.

[19] Dresselhaus MS, Jorio A, Hofmann M, Dresselhaus G, Saito R. Nano Lett 2010;10(3):751.

[20] Ago H, Kugler T, Cacialli F, Salaneck WR, Shaffer MSP, Windle AH, et al. J Phys Chem B 1999;103(38):8116-21.

[21] Grossiord N, Loos J, Van Laake L, Maugey M, Zakri C, Koning CE, et al. Adv Funct Mater 2008;18:3226-34.

[22] Hecht D, Hu L, Gruner G. Appl Phys Lett 2006;89:133112.

[23] Socher R, Krause B, Boldt R, Hermasch S, Wursche R, Pötschke P. Compos Sci Technol 2011;71:306-14.

[24] Socher R, Krause B, Müller MT, Boldt R, Pötschke P. Polymer 2012;53:495-504

[25] Zonder L, Ophir A, Kenig S, McCarthy S. Polymer 2011;52:5085-91.

[26] Bose S, Khare RA, Moldenaers P. Polymer 2010;51(5):975-93. 